\title{
A Framework to Support the 5G Densification
}

\author{
Eleni Theodoropoulou ${ }^{1(\bowtie)}$ (D), Ioanna Mesogiti ${ }^{1}$, \\ George Lyberopoulos ${ }^{1}$, George Kalfas ${ }^{2}$, Christos Vagionas ${ }^{2}$, \\ Nikos Pleros ${ }^{2}$, Annachiara Pagano ${ }^{3}$, Mauro Agus ${ }^{3}$, Luiz Anet Neto ${ }^{4}$, \\ Nikos Psaromanolakis ${ }^{5}$, and Athina Ropodi ${ }^{5}$ \\ ${ }^{1}$ COSMOTE Mobile Telecommunications S.A., 99 Kifissias Avenue, \\ 15124 Maroussi, Athens, Greece \\ etheodorop@cosmote.gr \\ 2 Aristotle University of Thessaloniki, Thessaloniki, Greece \\ 3 Telecom Italia, Turin, Italy \\ 4 Orange Labs, Lannion, France \\ 5 Incelligent, Athens, Greece
}

\begin{abstract}
G networks are shaping a new ecosystem necessitating various transformations of the existing network infrastructures combined with the use of network softwarization and programmability, so as to satisfy the needs of all the involved stakeholders (telecom/service providers, infrastructure owners, tenants, vertical industries, end-users, etc.), while a wide range of issues have to be addressed spanning from technology to business domains. The 5G-PPP project 5G-PHOS [1] proposes a novel framework to allow telecom operators and service providers to overcome $5 \mathrm{G}$ densification issues while supporting the stringent $5 \mathrm{G}$ requirements in a flexible and cost efficient manner to allow for commercialization. This paper aims at providing indicative architectural instantiations of the 5G-PHOS solution, depicting the way the technology supports the $5 \mathrm{G}$ requirements and the stakeholders' needs along with the functionalities and the deployment feasibility of an ambitious 5G fronthaul/backhaul network solution.
\end{abstract}

Keywords: $5 \mathrm{G} \cdot$ Use cases $\cdot 5 \mathrm{G}-\mathrm{PHOS}$ architecture $\cdot$ Architectural instantiations · Fiber-wireless · Optical technologies · R-RRH · SL-RRH · Massive MIMO $\cdot$ Flexbox

\section{Introduction}

The $5 \mathrm{G}$ vision to support the next generation services mandates the satisfaction of very strict user experience and system performance Key Performance Indicators (KPI) imposed by the 5G-PPP, that the current networks are incapable of satisfying without being transformed; including, among others, efficient feasible and affordable fronthaul/backhaul networks and related infrastructures to support the ultra-broadband 5G New Radio (NR) requirements [2-8]. In particular, the densification which is expected to be an integral part of $5 \mathrm{G}$ deployment, is about $10 \mathrm{x}$ denser than $4 \mathrm{G}$ and $100 \mathrm{x}$ denser than $3 \mathrm{G}$ networks and carries a number of issues to be addressed, such as the exploitation of higher frequency bands for extreme data throughput, low latency 
and ubiquitous coverage to be achieved, limited real-estate in urban and hotspot areas, the need for new regulations to facilitate the costly and time-consuming deployment of Base Stations/Small Cells and fibers, not to mention the hardware equipment costs associated with the Centralized Radio Access Network (C-RAN) deployment to address the expected explosion of the fronthaul (FH) capacity. A framework to help the telecom/service provider to mitigate and overcome the densification problems and deployment costs, is currently missing from the $5 \mathrm{G}$ solution pool, and is offered via the 5G-PHOS 5G-PPP EU project.

More specifically, the 5G-PHOS project aims at providing Mobile Network Operators (MNOs) and possibly, verticals and/or infrastructure owners (municipalities, stadium owners, etc.) [13], with an attractive fronthaul network solution, both in terms of performance and cost, especially addressing the 5G NR densification and $5 \mathrm{G}$ capacity issues. By capitalizing on existing wireless and optical technologies, the 5GPHOS transforms the current all-digital Point-to-Point (PtP) FH to a Point-to-MultiPoint (PtMP) digital and analog converged Fiber-Wireless (FiWi) FH, bridging one centralized to multiple remote locations through combined fiber and ultra-broadband wireless links, while maintaining compatibility with the standardized enhanced Common Public Radio Interface (eCPRI) - capable of combating the FH capacity explosion by supporting higher FH splits.

The paper is organized as follows: To start with, an overview of the 5G-PHOS project is presented to facilitate better understanding of the framework and the solution innovations, as well as the impact of 5G-PHOS solution on the $5 \mathrm{G}$ architectures. In the next section, the 5G-PHOS architecture is elaborated in the context of the three (3) Use Cases (UCs) envisioned by the project [12], to be followed by the architectural instantiations that depict the feasibility of the 5G-PHOS solution deployment so as to satisfy the needs and requirements of all the involved stakeholders, while conclusions are drawn at the end.

\section{5G-PHOS Solution Overview}

5G-PHOS addresses, in a flexible and efficient manner, the challenging 5G densification framework encompassing a range of urban, dense urban and hotspot environments, exhibiting different traffic density and coverage needs, that arise either under normal daily conditions or occasionally, during specific events (e.g., parades, outdoor concerts), while supporting the wide variety of 5G services, including: enhanced Mobile Broadband (eMBB), massive Machine-Type Communications (mMTC) aka Massive IoT (mIoT), Ultra-Reliable Low-Latency Communications (URLLC), and at the same time the Network Operation Services addressing the functional system requirements, including: flexible functions and capabilities, multi-tenancy, energy efficiency, interworking, security, etc. Indicative environments may span from typical residential and business areas in big European cities to highly touristic areas with seasonality based activity, and hotspots -usually small privately-owned establishments, like stadiums or concert halls hosting crowd events in a random or periodic frequency. In this context, the goal of the 5G-PHOS project is to develop novel 5G broadband architectures and evaluate them at dense, ultra-dense and hotspot areas. 
The main incentive behind the 5G-PHOS architecture is to create an ultrabroadband converged FiWi PtMP fronthaul network, capable of supporting the required 5G NR fronthaul bandwidth, while at the same time alleviating the need to install fiber terminations at every MNO Base Station (BS) site. Therefore, it comprises a very appealing solution for MNOs and infrastructure owner since it exhibit a wide range of benefits, such as:

- Utilization as front-/mid-/backhaul or Fixed-Wireless Access (FWA), meeting the 5G KPIs in terms of capacity, latency, QoS, energy efficiency, etc.

- Fast 5G network deployment capability (use of wireless (PtMP) vs. fibers)

- Cost efficiency (utilization of novel optical/wireless technologies, reliable wireless technologies, energy efficient components, etc.)

- Support of a wide range of UCs/scenarios over a single architecture

- Flexibility/versatility at various levels (e.g., wireless links at 5G networks' lastmile, flexible allocation of resources planned and handled by SDN, flexible channel/sub-band allocation framework to support different UCs)

- Scalability (modularity of the MIMO antennas and network reconfigurability to upscale in terms of capacity)

- Multi-tenancy, multi-operator, multi-domain support through slicing and infrastructure/resources sharing

- Interoperability with and smooth migration from 4G networks/solutions

The 5G-PHOS solution builds upon the prevalent enhanced eCPRI standard and creates the necessary infrastructure to interconnect eCPRI-capable equipment in a PtMP manner, that is, the centralized equipment, e.g. in the fronthaul case the Base Band Unit (BBU), can be concurrently connected to several Remote Radio Heads (RRHs), through a converged FiWi network, as depicted in Fig. 1.

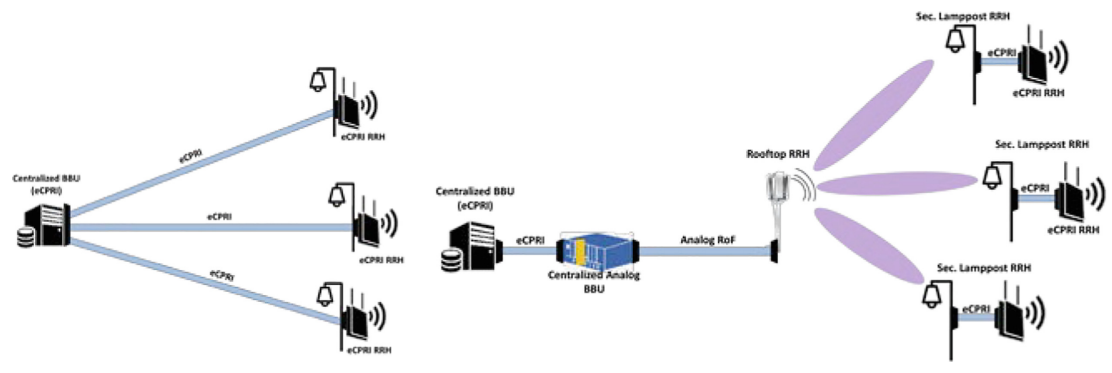

Fig. 1. 5G-PHOS transforms the PtP dedicated fiber link Fronthaul (left side) to a converged Fiber-Wireless PtMP high capacity analog Fronthaul (right side).

The 5G-PHOS aims to produce a powerful Photonic Integrated Chip (PIC) technology toolkit capitalizing on novelties in optical technologies, such as Indium Phosphide (InP) transceiver, Triplex optical beamformers and multi-bitrate optical communications into next generation fronthaul in order to migrate from CPRI-based to 
integrated FiWi packetized C-RAN fronthaul supporting Millimeter Wave (mmWave) massive Multiple-Input Multiple-Output (mMIMO) communications.

The novel technologies combined into one powerful suite and their associated benefits are the following:

- Analog Radio-over-Fiber (A-RoF) transmission, enabling the use of higher order modulation schemes, resulting in low complexity of Rooftop-RRHs and high spectral efficiency.

- Synergy of mmWave wireless radio and mMIMO antennas to provide increased capacity and link reliability, while reducing the need for PtP fiber deployment.

- Combination of Optical Beamforming Networks (OBFNs) and massive MIMO mmWave antennas, enabling the creation of complex Rooftop RRH antenna patterns to reach the multitude of lamppost antennas with highly-directed beams; thus enhancing the flexibility of the wireless links.

- Cutting-edge PIC technologies for a number of critical functionalities, i.e., laser arrays, Optical Beamforming Networks (OBFNs), Reconfigurable Optical Add Drop Multiplexers (ROADMs), offering high-power, low-cost, better powercoupling, high-responsivity, etc.

- Ethernet-based network processors allowing not only for connectivity/ integration with (MNO) equipment for a smooth $4 \mathrm{G}$ to $5 \mathrm{G}$ migration, but also enabling the Software Defined Networking (SDN) framework for efficient network management towards low-latency and energy-efficient reconfigurable 5G network-on-demand schemes.

In the end, 5G-PHOS expects to release a seamless, interoperable, Radio Access Technology (RAT)-agnostic and SDN-programmable high-capacity FiWi 5G C-RAN network solution for dense, ultra-dense and hotspot scenarios supporting 64x64 MIMO antennas in the V-band able to offer data rates up to $400 \mathrm{~Gb} / \mathrm{s}$, while efficiently facing the CPRI fronthaul capacity problem.

\section{5G-PHOS Architecture for Representative Use Cases}

The 5G-PHOS solution is versatile and can be used not only as fronthaul but also as midhaul or backhaul, by appropriate placement of the 5G-PHOS stack within 3GPP's three-way split defining the respective entities:

- the Centralized Unit (CU) located at the MNO's Central Office (backhaul)

- the Distributed Unit (DU) usually at the basement of the building containing the antennas or somewhere close-by (fronthaul) and

- the Radio Unit (RU) including the radio elements and the unit closest to the mobile users (fronthaul).

The 5G-PHOS proposes 3 variants (Fig. 2, Fig. 3, Fig. 4) of the architectural design, one for each of the 3 UCs envisioned by the project: the dense, ultra-dense and hotspot $[9,10]$. 


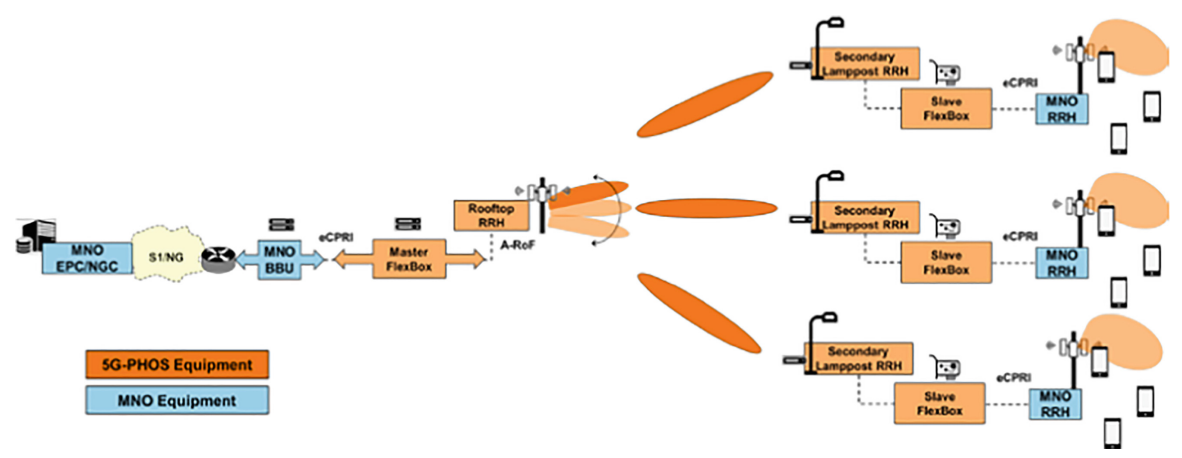

Fig. 2. The 5G-PHOS PtMP fronthaul solution for Dense Area Use Case. (Color figure online)

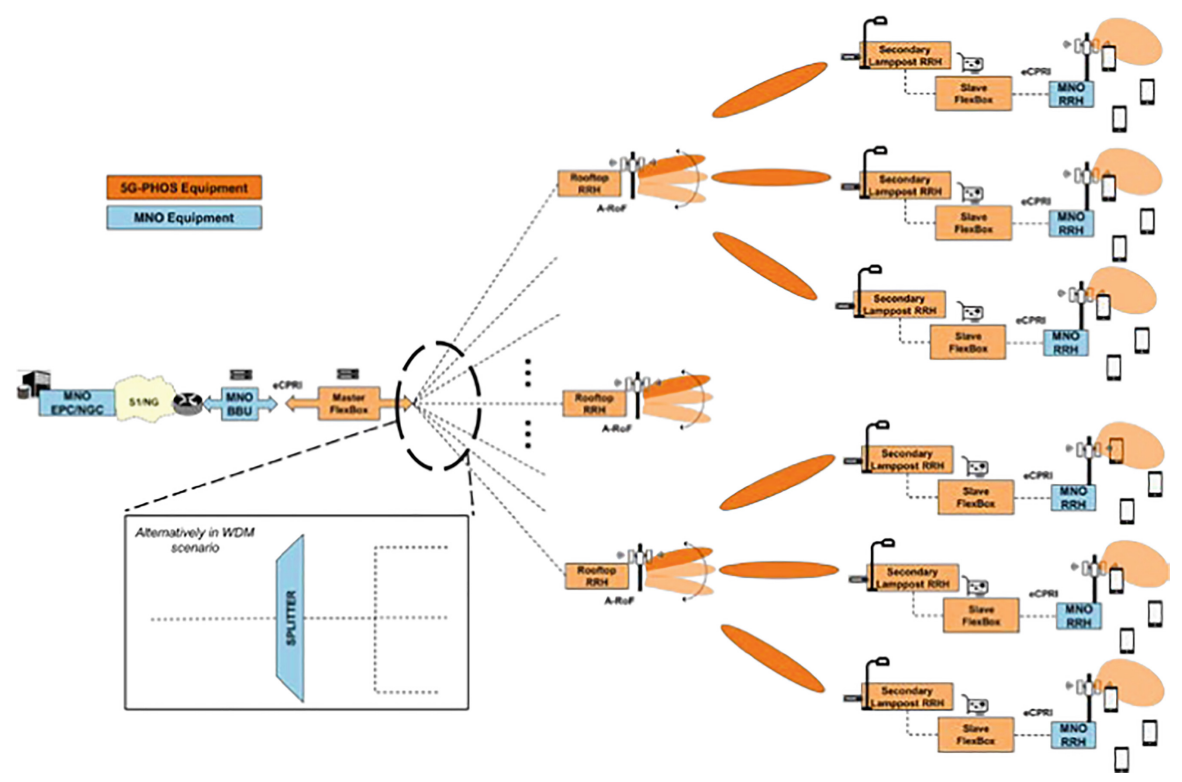

Fig. 3. The 5G-PHOS PtMP fronthaul solution in the Ultra-Dense Use Case. (Color figure online)

The 5G-PHOS architecture (as shown in Fig. 2, Fig. 3, Fig. 4) introduces a number of nodes (in orange) the functionality of which is described below:

- Master Flexbox: It receives the digital eCPRI signals generated by the BBU/CU/DU, and loads the incoming Ethernet packets onto analog carriers to traverse the converged FiWi network. It creates and transmits all FH control plane signals and hosts all higher-level operations such as SDN agents and access control, while creating the analog signals for the downlink direction. Regarding SDN functionalities, the Master Flexbox supports OpenvSwitch (OvS), a production quality, multilayer virtual switch, while OpenFlow, OVSDB and NETCONF 


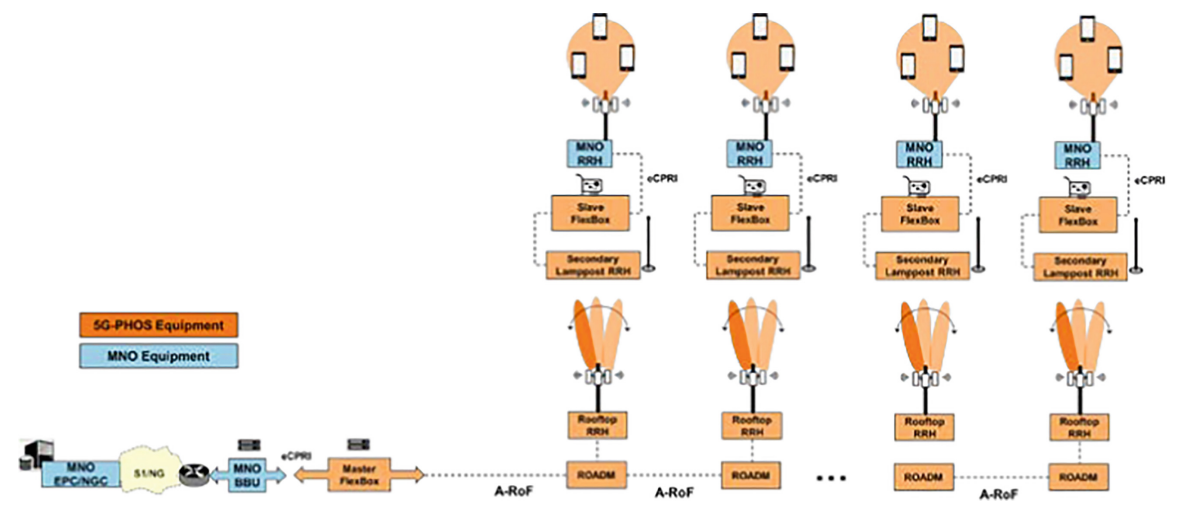

Fig. 4. The 5G-PHOS PtMP fronthaul solution in the Hotspot area Use Case. (Color figure online)

protocols support the interaction with the SDN controller [11]. In terms of hardware, the Master Flexbox contains the network controller that hosts the upper-layer functionalities and Ethernet packet processing, as well as the FPGA module that performs the Digital Signal Processing (DSP) functions and the transceivers of the actual data. The Master Flexbox is connected through an Ethernet port (provided by the network controller) to the centralized unit (BBU, CU, DU or $4 \mathrm{G} / 5 \mathrm{G}$ core depending on the 5G-PHOS stack placement).

- Rooftop RRH (R-RRH): It receives the analog signals from the Master Flexbox and transmits them over the air in the mmWave band. In terms of hardware, it contains the OBFN module and the mmWave massive MIMO antenna. In the hotspot UC (Fig. 4), the R-RRH contains also the ROADM module to perform wavelength selection among multiple wavelengths employed.

- Secondary Lamppost RRH (SL-RRH): It comprises the mmWave antenna that receives the signals from or transmits to the R-RRH. It is simpler than the R-RRH, since it communicates to a single R-RRH only in the uplink direction and does not contain OBFNs.

- Slave Flexbox: It contains a lightweight version of the network controller and an FPGA that receives and decodes the signals belonging to the FH Control and Data planes (CP, DP). Moreover, the Slave Flexbox hosts some high-level operations such as SDN agents, while it creates the analog signals for the uplink direction for both CP and DP. It is connected through an Ethernet port (provided by the network controller) to the remote unit (RU, DU, or gNB depending on the placement of the solution). Again, the Slave Flexbox supports OpenvSwitch and hence OpenFlow, OVSDB and NETCONF.

In the variant of the ultra-dense UC architecture, the number of R-RRH (as well as MNO RRH) modules in a geographical area may increase up to 16 R-RRH connected to the Master Flexbox by either 16 fibers or alternatively by the use of 16 wavelengths in a WDM manner. In this way, MNOs or Infrastructure Owners (IOs) can multiply accordingly the offered capacity in areas with great population densities and increased capacity requirements, such as city centers. 
What differentiates the hotspot architecture is the adoption of a WDM approach to increase capacity, supported by the employment of the 5G-PHOS ROADM module placed in a single fiber bus topology, recommended for internal cabling.

\section{5G-PHOS Architectural Instantiations}

This Section presents the architectural instantiations that fulfill the requirements of the main stakeholders of the 5G-PHOS solution (that is, MNOs, site owners, tenants) in a flexible and economically viable way, in dense, ultra-dense, hotspot areas, based on representative scenarios: multi-operator through slicing (sharing part or the entire 5GPHOS solution), multi-tenancy to support multiple verticals concurrently, multidomain operation (e.g., fixed and wireless belonging to the same or different operators).

\section{Scenario \#1: Multi-operator and slicing support of "local" Operator(s)}

Operator A shares with Operator B (having no or limited "presence" in the area), part of or the entire 5G-PHOS solution (R-RRHs, SL-RRHs, 5G equipment) already deployed to meet Operator A own capacity, coverage and (own) customer needs. Two (2) sub-scenarios can be considered:

\section{(a) Operator B as Operator's A tenant sharing the same SL-RRH}

Operator B connects its antennas directly (through the Ethernet interface) to an existing SL-RRH that belongs to Operator A, as depicted in Fig. 5. The SDN framework allows for slicing functionality support and provides the Operator A with the capability of forming a "slice" for Operator B with several options based on rules, such as: (a) allocate dedicated sub-bands to Operator B either a static (pre-defined) way or dynamically with an upper limit (scaled-up or scaled-down automatically based on optimization algorithms that are running on the SDN controller) and b) allocate certain capacity within an existing sub-band, for instance share 1 Gbps out of a 2.4 Gbps subband. The rules are transmitted to both the Master and Slave Flexbox units by use of the OpenFlow and OVSDB protocols (based on TCP/IP).

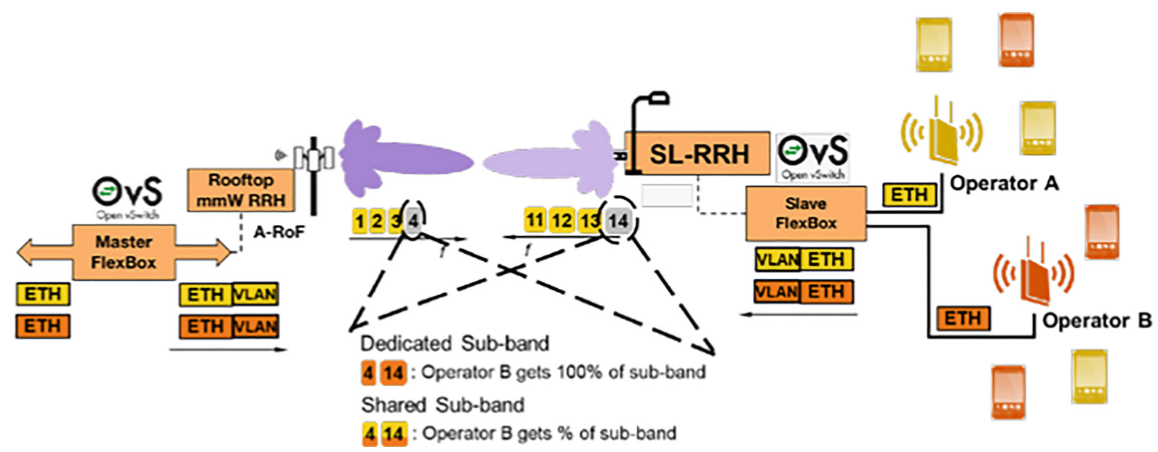

Fig. 5. 5G-PHOS multi-operator architectural instantiation using the same SL-RRH. 


\section{(b) Operator B as Operator's A tenant sharing the same R-RRHs}

Operator B deploys own SL-RRHs and 5G RRHs (connected to its SL-RRH through the Ethernet interface provided by the Slave Flexbox unit), as depicted in Fig. 6. This new SL-RRH communicates wirelessly to the R-RRH owned by Operator A, and is added to the 5G-PHOS system. Again, Operator A forms a separate "slice" for Operator B through the SDN (assign sub-bands static or dynamic, limit traffic bandwidth, increase/decrease capacity if the contract changes, etc.)

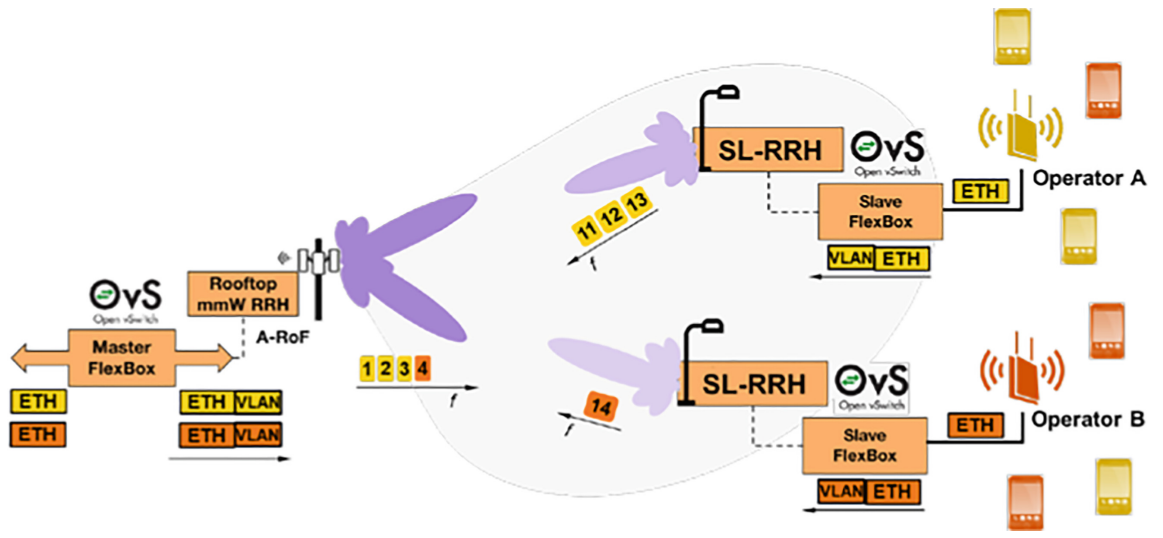

Fig. 6. 5G-PHOS multi-operator architectural instantiation using different SL-RRHs.

\section{Scenario \#2: MNO adopts 5G-PHOS solution at dense/ultra-dense areas}

The Operator/MNO deploys the 5G-PHOS solution (R-RRHs, SL-RRHs, 5G equipment) to meet its own needs (mobile and fixed subscribers via fixed wireless access (FWA), tenants, excluding "local" MNOs) in a dense/ultra-dense area in a flexible and economically viable way. Two (2) sub-scenarios can be considered:

\section{(a) Multi-tenancy to support multiple verticals concurrently through slicing}

A vertical application, e.g., based on security cameras can be easily integrated into the 5G-PHOS system, since they are connected to the MNO's SL-RRH through the Slave Flexbox's Ethernet interface as depicted in Fig. 7. As in Scenario \#1, the cameras' IP addresses are utilized for the assignment of fronthaul capacity to them through the OpenvSwitch interface. In case that more than one cameras are connected to the same SL-RRH, the SDN rules could be either port-based (the same rule for the total traffic) or IP-based depending on camera/user requirements. As described in Scenario \#1, the security cameras can either get dedicated sub-bands or share sub-bands with other services, while the OpenvSwitch ensures that the traffic will not exceed the allocated capacity. 


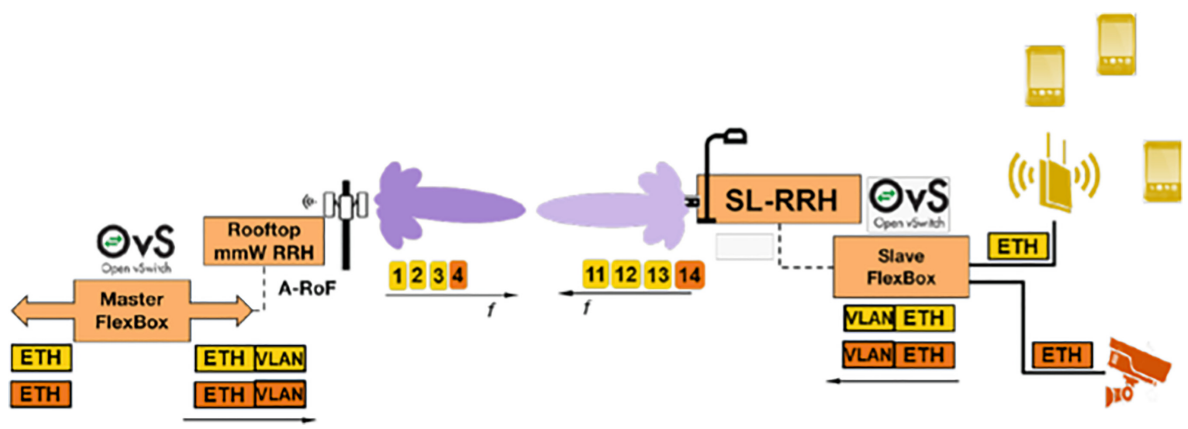

Fig. 7. 5G-PHOS multi-tenancy architectural instantiation using the same SL-RRH.

a)

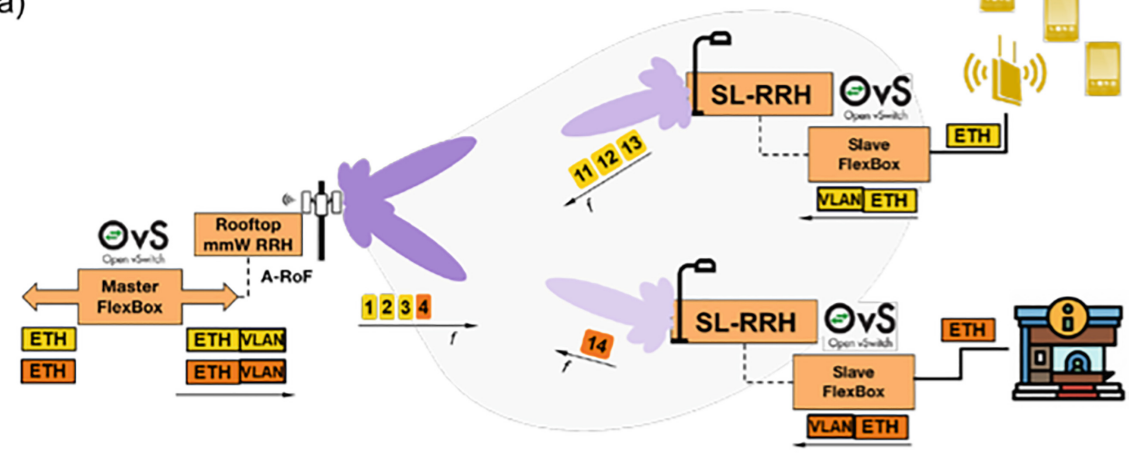

b)

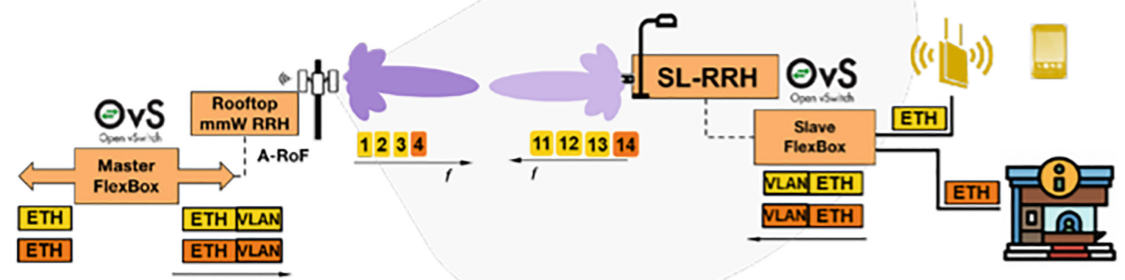

Fig. 8. 5G-PHOS multi-domain architectural instantiation a) using dedicated SL-RRHs and b) sharing the same SL-RRH.

\section{(b) Support of multi-domain applications}

The MNO uses the 5G-PHOS solution to provide fixed access to a subscriber, for instance a Tourist Information kiosk in a central square. Two alternatives can be envisaged/supported: 
(i) Through a dedicated SL-RRH (that will serve the kiosk) (Fig. 8 (a)) added to the $5 \mathrm{G}-\mathrm{PHOS}$ system and managed by the MNO via the SDN framework

(ii) Direct connection of the kiosk router/switch to an existing SL-RRH (Fig. 8 (b)). The kiosk becomes a tenant connected to the 5G-PHOS solution (see Multitenancy to support multiple tenants/verticals concurrently through slicing).

Scenario \#3: 5G Infrastructure Owner (IO) serves own and tenant needs supporting Multi-tenancy and Multi-operator domain

The infrastructure owner (e.g., a stadium owner) has deployed its own 5G-PHOS solution for its own needs (own staff, fans). In addition, it offers the possibility to verticals and MNOs to deploy their own infrastructure.

The stadium owner has set up its own network consisting of a series of R-RRHs (deployed in bus topology) connecting several SL-RRHs (spread out in the fans' seating area) offering access to WiFi access points through its Slave Flexbox Ethernet interfaces. During a football match -it is necessary or a contractual obligation with third parties- to deploy extra services for various tenants, such as MNOs, TV broadcasters or first-responders. The tenant's equipment, as depicted in Fig. 9 could be installed: (i) either directly to the Ethernet interface of an already deployed SL-RRH or the IO could allow the tenant -upon agreement- to deploy its own SL-RRH(s).

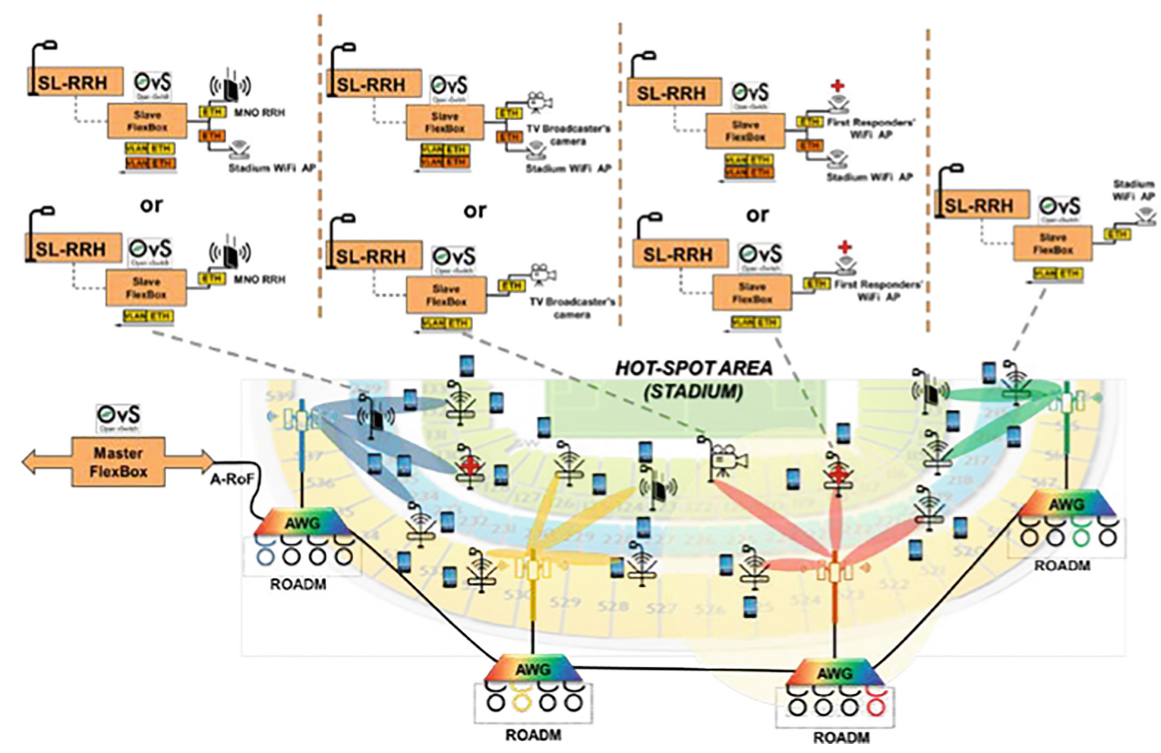

Fig. 9. 5G-PHOS Infrastructure Owner supporting multi-tenancy \& multi-operator schemes.

Since the IO is the 5G-PHOS solution owner, the IO has exclusive access to the SDN framework and thus can manage the various "slices" flexibly. For instance, TV broadcaster's camera equipment connected to a dedicated or shared SL-RRH can be assigned to a static and dedicated "slice" with the necessary number of sub-bands, since 
the cameras produce a constant load and it is critical not to disrupt the service. The first responders' dedicated WiFi APs can either have a dedicated sub-band or guaranteed capacity within a shared sub-band, since the service is critical, not high-load and used in emergency situations only. The MNOs can also lease (static or dynamic) capacity for the duration of the football game.

\section{Discussion}

This paper focuses on the 5G-PHOS solution and its impact on the $5 \mathrm{G}$ networks architecture, especially addressing the $5 \mathrm{G}$ densification. The 5G-PHOS solution is a high-capacity FiWi 5G C-RAN network solution for dense, ultra-dense and hotspot scenarios supporting $64 \times 64$ MIMO antennas in the V-band able to offer data rates up to $400 \mathrm{~Gb} / \mathrm{s}$, while efficiently facing the CPRI fronthaul capacity problem. Depending on its placement in the MNO's network, the 5G-PHOS solution can be utilized as telecom bridge for fronthaul, midhaul, backhaul, or combination of them, as well as a FWA solution and WiFi hotspot solution. Focusing on the fronthaul, it comprises an attractive network solution, in terms of performance cost and supported functionalities, for the involved stakeholders (e.g., telecom operators/MNOs, vertical infrastructure owners), while alleviating the need for fiber deployment at every BS site. The paper elaborates on the 5G-PHOS through architectural instantiations, explaining how the 5G user and system requirements as well as the involved stakeholders' needs are supported by the 5G-PHOS solution.

Acknowledgements. The research leading to these results has received funding from the European Union's Framework Programme Horizon 2020 under grant agreement No. 761989 and project name "5G-PHOS: 5G integrated Fiber-Wireless networks exploiting existing photonic technologies for high-density SDN programmable network architectures".

\section{References}

1. 5G-PHOS H2020 5G-PPP Project. http://www5g-phos.eu/

2. 5G-PPP Program, European Commission, Living document on "5G-PPP use cases and performance evaluation models"

3. 5G-PPP, The 5G Infrastructure Public Private Partnership. https://5g-ppp.eu/

4. 5G Americas: 5G Services and Use Cases, White paper, November 2017. http://www. 5gamericas.org/files/6214/3569/1603/4G_Americas_Mobile_Broadband_Evolution_ Toward_5G-Rel-12_Rel-13_June_2015.pdf

5. Nokia white paper: Ultra dense networks (2016)

6. Osseiran, A., Monserrat, J., Marsch, P. (eds.): 5G Mobile and Wireless Communications Technology. Cambridge University Press, Cambridge (2016)

7. 5G-PPP View on 5G Architecture, Version 2.0 (2017). https://5g-ppp.eu/wp-content/ uploads/2018/01/5G-PPP-5G-Architecture-White-Paper-Jan-2018-v2.0.pdf

8. 3GPP, TS 23.501: System Architecture for the 5G System; Stage 2. Rel. 15

9. 5G-PHOS, Deliverable D2.1: Initial report on use cases, system requirements, KPIs and Network Architecture. http://5g-phos.eu/pdf/5G-PHOS_D2.1_Final.pdf 
10. 5G-PHOS, Deliverable D2.2: Initial design of 5G-PHOS flexbox and RRH system architecture and component specifications including resource allocation and SDN functions, (Internal to the consortium)

11. 5G-PHOS, Deliverable D7.1: SDN controller and FPGA-based DSP engine implementation (Confidential)

12. Lyberopoulos, G., et al.: Fiber-wireless Fronthaul/Backhaul network architectures for 5G. In: Proceedings of the Computer Aided Modeling and Design of Communication Links and Networks (CAMAD), 2018 IEEE 20th International Workshop, Barcelona, Spain, 17-19 September 2018. https://doi.org/10.1109/camad.2018.8514991

13. Mesogiti, I., et al.: A framework to support the role of telecommunication service providers in evolving 5G business models. In: MacIntyre, J., Maglogiannis, I., Iliadis, L., Pimenidis, E. (eds.) AIAI 2019. IAICT, vol. 560, pp. 60-69. Springer, Cham (2019). https://doi.org/10. 1007/978-3-030-19909-8_5 